\title{
Medical Monitoring Applications for Wearable Computing
}

\author{
DEJAN RASKOVIC ${ }^{1}$, ThOMAS MARTIN ${ }^{2}$ AND EMIL JOVANOV ${ }^{3}$ \\ ${ }^{1}$ Department of Electrical and Computer Engineering, The University of Alaska Fairbanks, \\ ${ }^{2}$ The Bradley Department of Electrical and Computer Engineering, Virginia Tech, \\ ${ }^{3}$ Department of Electrical and Computer Engineering, The University of Alabama in Huntsville \\ Email: d.raskovic@uaf.edu
}

\begin{abstract}
Medical monitors have benefited from technological advances in the field of wireless communication, processing and power sources. These advances have made possible miniaturization and prolonged operating times of medical monitors, as well as their global integration into telemedical systems. This allows patients to have real-time feedback about medical conditions while going about their normal daily activities. System designers are facing specific issues related to monitor acceptability, application requirements, power consumption and system connectivity. In this paper we discuss system design issues, present a survey of existing systems and sensors, and introduce two taxonomies of medical monitoring applications for wearable computing.
\end{abstract}

\section{INTRODUCTION}

Wearable computing covers a broad range of devices and applications [1]. An increasingly important application of wearable computers is as an intelligent medical monitoring device providing real-time feedback to patients, athletes during training and healthy users about their physiological state. A patient can wear the device during normal daily activity, allowing medical staff to obtain a much clearer view of the patient's condition than is available from short periods of monitoring in the hospital or doctor's office [2]. Note that the intelligent medical monitoring device is to be worn and used by a patient, not by medical personnel, e.g. doctors, nurses and emergency medical technicians [3]. It should also be distinguished from medical data acquisition devices currently available, which have little or no real-time processing, limiting their functionality to recording signals that will later be processed off-line $[4,5]$.

The major obstacles to wider use of intelligent wearable medical monitors include size and weight of the monitor, sensor implementation and connectivity [6]. The present generation of CPUs has sufficient performance and sufficiently low power consumption to process the signals in real-time, increasing the intelligence of medical sensors. Technological advances in application specific integrated circuits (ASICs), battery capacity and wireless technology have resulted in reduced size of medical sensors and systems, the ability to communicate wirelessly, and the ability to operate on batteries for prolonged periods of time. This combination of high performance, low power and wireless communication allows prediction of impending medical emergencies and feedback about current user physiological status while users go about their normal routine. For example, a wearable device could be used to monitor the recovery of heart rate after exercise, which has been shown to be useful as a prognostic tool [7]. A wearable device could also be useful for low intensity monitoring in the case of elderly patients; it has been shown that such monitoring can reduce costs of hospitalization and care for heart failure patients [8]. In order to be most useful to the patient, the device must be designed properly in terms of size, weight, performance, reliability and privacy. This paper describes design issues that are specific to medical monitoring applications and then presents two taxonomies. The first taxonomy classifies systems based on their capability and wearability. The second one categorizes systems based on locality of users and data access points.

The remainder of the paper is organized as follows. Section 2 describes the differences between medical monitoring applications and other wearable computing applications, and some of the difficulties that lie in the way of their wider acceptance. Section 3 presents a brief survey of medical monitoring devices and systems, showing that medical monitoring is rich in possible applications for wearable computing. Section 4 presents two taxonomies of personal medical devices that classify them according to various parameters of interest. Finally, Section 5 presents our conclusions and future work.

\section{ISSUES IN WEARABLE MEDICAL APPLICATIONS}

Medical monitoring applications of wearable computing differ from general-purpose wearable computing applications in several respects, in degree if not in kind. First, user interaction tends to be much more limited. The interaction often consists only of warnings issued to the user and notes and settings entered by the user. Second, the performance/power 
trade-off rules change with the change of environment or user condition. When operating under normal conditions, low power consumption is usually the primary concern for such devices, while in emergencies the performance becomes more important. Third, there is an emphasis on signal processing that does not exist to the same degree in general purpose wearable computing applications. Typically, the sampling range is relatively low, from a few Hertz to a few hundreds of Hertz, but reliability must be high. In this respect, wearable medical monitoring applications more closely resemble scientific computing applications than other wearable applications. Finally, the wearable medical computer faces more stringent requirements in terms of privacy, security, reliability, government regulation and the manufacturer's legal responsibility.

\subsection{Applications and obstacles}

It is reasonable to expect that many types of medical monitoring that currently require the patient to visit a hospital or doctor's office and be connected to a tethered machine can eventually be performed using a wearable device. However, in the near term, we see the following applications:

- Monitoring of myocardial ischemia [4, 9, 10, 11].

- Epileptic seizure detection [12].

- Drowsiness detection (alertness monitoring) [13, 14].

- Physical therapy feedback, such as for stroke victim rehabilitation $[15,16]$.

- Long-term monitoring for circadian rhythm analysis of heart rate variability (HRV) [2, 17].

- Sleep apnea monitoring [18].

- Stress level assessment and stress resistance prediction [19].

The major obstacles to wider use of intelligent wearable medical monitors include the following:

- Existing wet sensors are impractical and tend to irritate the skin after prolonged periods of use. (Wet sensors are those that use a gel to improve contact with the skin.)

- Contact resistance between the electrode and skin changes over time. For example, gels that are used to improve contact in wet sensors dry out over time. Sensors can temporarily or permanently lose contact with the skin as the user moves about.

- Size and weight of intelligent devices. Available data acquisition Holter monitors (portable electrocardiographs) are typically the size of a Walkman ${ }^{\mathrm{TM}}$, which is larger and heavier than desirable, especially for prolonged monitoring during normal activity. Device intelligence in addition to data storage further increases size and weight.

- Routing and connection of sensors. Most medical monitoring applications require a large number of analog input channels, each with their own particular requirements in terms of signal range, amplification and filtering. For example, up to a dozen channels are needed for sleep studies (EEG/EMG/EOG). Using wired connections for all these sensors is impractical in everyday settings, because the wires will interfere with the user's movement, be unsightly and tangle with objects in the environment.

- Regulation by government medical agencies, such as the Food and Drug Administration in the United States.

- Responsibility for outcome of medical conditions, i.e. a manufacturer will want to minimize the chance of being sued if the device does not correctly predict a medical event. It is difficult to determine if there was a malfunction of device, a lack of knowledge of other conditions of the patient or whether the lawsuit was justified.

As noted above, security, privacy and reliability are particularly important features for wearable medical monitoring devices:

- Security. The main security issue with wearable medical monitoring applications is how not to disclose the information collected about the user, rather than an issue of what information about others the user is allowed to record or access [20]. In this respect, the wearable medical monitoring applications are somewhat at an advantage over other wearable applications, which likely have a great number of societal and legal barriers to overcome [20]. Since the user would like the data collected to remain private, the data should be encrypted and made physically secure. One issue of the encryption is that it must balance strength of encryption with the available power (both in terms of Watts and MIPS). It would be undesirable to use an encryption mechanism that is only slightly more secure while requiring a great deal more performance and power.

- Privacy. The one privacy issue that medical monitoring applications raise that other wearable computing applications do not is that simply wearing the device may disclose information that the user would like to keep private, as wearing the device may disclose to the user's employer/insurer/acquaintances that the user is suffering from a medical condition. While this particular issue is shared with other medical equipment, so far as we know, no one has raised this as an issue in wearable computing. Such disclosure requires that the wearable monitoring device be as unobtrusive as possible. The larger and bulkier the device, the more likely it is to be observed by those around the user. Thus, a proper balance of power and performance is desirable even from the standpoint of privacy. From that standpoint, it is clear that wireless sensors are crucial for privacy.

- Reliability. As for reliability, medical wearable electronics must be more reliable than their general-purpose counterparts. A wearable ECG must function for as long as expected (i.e. for its full estimated battery life, usually $48-72 \mathrm{~h}$ for commonly used technology) without any failures, otherwise its purpose is defeated. Moreover, the device should allow recovery of data collected prior to the time when the batteries unexpectedly ran out. For wearable medical electronics that are intended to give advance warning of serious conditions, a failure could be life threatening. 
Finally, we envision a collection of wearable medical sensors, communicating with a central processing element, also wearable, which processes the data in real-time and provides information to the user about medical status [12], akin to Gordon Bell's body network and 'guardian angel' [21].

This 'guardian angel' could be networked into a wireless telemedical network, so that specialized medical response services could be contacted in the event of a medical emergency. Alternatively, if an episode is not life threatening but still requires intervention, the medical service provider could be contacted and data could be uploaded [12]. The user or the caregiver or both could formulate triggers that cause even more data to be collected, additional sensors to be enabled or medical personnel to be contacted.

\subsection{Smart clothes}

Wireless communication as an unobtrusive way of sensor networking has an alternative in smart clothes. This approach can significantly decrease the price of sensors, but might prove to be inferior when new sensors have to be integrated into a Body Area Network.

Several groups currently work on smart clothes. University of Rochester Center for Future Health [22] developed and patented smart socks that detect circulation problems of diabetics and smart bandages that bind to specific DNA sequences of different bacteria and emit detectable light when bacteria are detected. Georgia Institute of Technology, School of Textile and Fiber Engineering [23] with the Sensatex company developed Smart Shirt [24]. Sensatex plans to market a Smart Shirt System that will incorporate technology advances in smart clothes, wearable computing and wireless communication into a product that will allow data collection and monitoring of biometric information. The Electronic Textiles group at Virginia Tech is developing fabrics that have interconnections and components woven into them, which can be used for both wearable and nonwearable applications $[25,26]$. Infineon has a prototype of an MP3 player integrated into clothing [27].

\subsection{Implantable sensors}

Designers of intelligent implanted sensors are facing more stringent requirements regarding size, power consumption and power efficient communication. The choice of a communication frequency dictates the size of the antenna and the whole sensor. In addition, reliable transfer of data from the implanted sensors is one of the most important tasks researchers are facing. Low power consumption is crucial in the case of implantable sensors, because it is highly desirable either to ensure very long-life battery operation or to design an implantable device that could be inductively or optically powered/recharged.

\section{SURVEY}

This section provides some examples of wearable computing in personal medical applications. Some of the devices and systems presented here fall into the category of wearable computers, while the others are presented either for contrast, historical reasons, or because the authors believe that they can be improved and incorporated into future wearable medical computing systems. Systems are grouped according to the medical condition to which they apply or could apply. We present both commercial and research projects in the field of personal intelligent monitors. Although wireless telemetry has been available for a few decades, wireless intelligent sensors capable of real-time signal processing have become technologically feasible only recently. Therefore, most wireless intelligent sensors available in the open literature reviewed in this section represent research projects. Each example has a label that will be used in Section 4 to display its position in our taxonomy.

\subsection{Cardiopulmonary monitoring}

C1. Medtronic offers the Insertable Loop Recorder [5, 28], developed in collaboration with the Division of Cardiology, University of Western Ontario. It provides up to 14 months of monitoring and data acquisition of critical cardiac events. Up to 40 min of history can be stored after an episode. This device weighs $17 \mathrm{~g}$, with an approximate volume of $8 \mathrm{ml}$. A previously recorded episode can be uploaded on demand to the computer for analysis.

C2. An implantable MEMS blood pressure sensor from CardioMEMS [29] was originally developed at Georgia Tech, for operation inside jet engines. The device can be implanted via a catheter into the heart's right ventricle and will take regular heart pressure readings. Readings can be transmitted from the sensor to a handheld computer.

C3. Meridian's TelePulse oximeter [30] measures the levels of oxygen in the blood, as well as the heart rate. The continuous readings are automatically transmitted over telephone lines to a telemedical server for analysis. The device consists of a probe that attaches to the patient's finger, and a miniature or handheld computer to process the data.

C4. The Seiko Pulsegraph [31] displays measurements of pulse rate and exercise history using a finger-mounted optical sensor and an acceleration sensor. The device employs a newly developed twin-FFT pulse analysis system and a bluelight LED sensor (instead of a usual red-light one) to solve the detection problems arising because of sensor movements and external light.

C5. Scientists at the d'Arbeloff Laboratory for Information Systems and Technology, MIT, have developed a Ring Sensor that continuously monitors heartbeat rate using a photoplethysmograph (PPG) signal and sends data wirelessly to a host computer [32]. The device is worn as a finger ring.

C6. Researchers from two Portugese universities [33] developed the Subcutaneous Implantable Capsule that uses phonocardiography as a method to monitor changes in heart 
rate. The capsule consists of a bimorph piezoelectric sensor, an analog signal processing module, a frequency modulated transmitter $(30 \mathrm{MHz}), 3.6 \mathrm{~V}$ battery and an antenna. Battery life was measured to be 30 days and the total size of the device is $75 \mathrm{~mm} \times 17 \mathrm{~mm}$.

C7. We developed a system for stress level assessment based on heart rate variability measurements [19]. The system performs synchronous measurement of the heart rate of each individual within the group, during prolonged stressful training. Data are stored locally (for up to $60 \mathrm{~h}$ ) and collected wirelessly from the group using mobile gateways.

\subsection{Diabetes control}

D1. A typical example of a commercial system is the Symphony ${ }^{\mathrm{TM}}$ Diabetes Management System [34] from Sontra Medical Corporation. Sontra offers a patch sensor that will continuously extract interstitial fluid, draw the analytes into the sensor, and measure and calculate the blood glucose concentration. The results are calculated and wirelessly sent to the receiver every $3.8 \mathrm{~s}$. Currently, the system is used for glucose measurements only, but the company plans to add sensors to measure other analytes as well.

D2. The 'skin breakdown detection' device is intended for people suffering from diabetes [35]. The device is worn in the shoe, and records temperature, pressure and humidity under the heel and metatarsal heads. The values are evaluated off-line periodically to detect abnormal stresses that lead to skin breakdown, which in a diabetes patient may necessitate amputation of the limb.

D3. Permanent implantable sensors are very promising for continuous monitoring of blood glucose levels [36]. Animas Corp. is developing a long-term (multi-year) implantable optical sensor, which will provide continuous readings of blood glucose and wireless communication with an accessory device [37].

\subsection{Brain and muscle activity recording/stimulation}

B1. Researchers from the University of Washington, Caltech and Case Western Reserve University have developed a miniature implantable microcomputer [38], capable of recording nerve and muscle signals from small animals during their normal activity. They use flexible metallic needles to collect signals from nerve bundles and micromachined silicon probes to record the activity of neural assemblies. However, to collect signals from individual neurons, scientists from the University of Washington are working on silicone MEMS probes that will mimic the performance of glass capillaries used on constrained animals. The implantable device consists of variable-gain amplifiers, a system-on-a-chip microcontroller and a highdensity memory. In this project, researchers decided to avoid antennas and charge pumps needed for RF-powered devices, due to size constraints of the implantable device. Instead, they plan to employ thin-film batteries.
B2. At the University of Michigan's Center for Wireless Integrated Microsystems, researchers developed a BiCMOS wireless stimulator chip [39, 40] that will be used in conjunction with micromachined passive stimulating microprobes. These probes were developed at the same university and are in regular medical use. This will allow wireless and stand-alone operation for an unlimited time period, since the chip uses a $4 \mathrm{MHz}$ carrier to receive both data and power through inductive coupling. The total power dissipation of the chip is less than $10 \mathrm{~mW}$ and the area it occupies is about $13 \mathrm{~mm}^{2}$.

B3. Another example of an RF-powered intelligent sensor is a miniature implantable wireless neural recording device [41] developed at the University of California, Los Angeles. This device records and transmits neural signals. The device size is less than $1 \mathrm{~cm}^{2}$ and power dissipation is measured at $13.8 \mathrm{~mW}$. Tests have shown that the transmitting range is up to $0.5 \mathrm{~m}$, and that the demodulated signal is highly correlated with the original signals in the range between $5 \mathrm{mV}$ and $1.5 \mathrm{mV}$.

\subsection{Swallowable and endoscopic pills}

P1. Researchers from the Universities of Glasgow, Edinburgh and Strathclyde are developing a capsule [42] traversing the gastrointestinal tract (part of IDEASIntegrated Diagnostics for Environmental and Analytical Systems project). The sensor gathers data that cannot be collected using traditional medical endoscopy. The device is battery-powered and integrates sensors, processing and RF bi-directional communication onto a single piece of silicon (current device size is $32 \mathrm{~mm} \times 11.5 \mathrm{~mm}$ ). This device uses a spread-spectrum based communication subsystem [43].

$P 2$. Given Imaging offers a commercially available system, the Given ${ }^{\circledR}$ Diagnostic System [44, 45]. A disposable imaging capsule M2A $(11 \mathrm{~mm} \times 26 \mathrm{~mm})$ passes through the gastrointestinal tract and wirelessly streams video images to the receiver worn on a belt. The signal is received through an array of antennas, which are also used to determine the exact location of the capsule [46, 47].

P3. The National Aeronautics and Space Administration (NASA) is developing the Fetal Monitoring System as a part of their Sensors 2000! program. This is a pill-shaped biotelemeter, used originally for space flight applications. New pills will be used to monitor the health of fetuses recovering from in-utero surgical repairs of life-threatening birth defects [48]. Currently, two different sensor pills are being developed-one measures pressure and temperature, while the other one measures $\mathrm{pH}$ and temperature. Plans exist for other pills that will measure the heartbeat rate, ECG, glucose, blood gasses etc. Pills are introduced during endoscopic fetal surgery and transmit data at 174-214 MHz. Low-power operation is achieved by low data rate (a pair of RF bursts at a frequency of about $1-5 \mathrm{~Hz}$ is transmitted) and by using a Pulse Interval Modulation (PIM) encoding 
scheme. Under these conditions (not typical for ECG signals), the expected transmitter lifetime is several months.

\subsection{Post surgery monitoring}

S1. The Oak Ridge National Laboratory is working on an implantable sensor that will allow non-invasive assessment of blood flow quality for the newly transplanted organs. The implantable unit, about the diameter of a quarter, will provide real-time information by transmitting tissue circulation data, assessed using optical sensors, to a nearby receiver [49].

S2. Researchers at the Biomechanics-Laboratory, OskarHelene-Heim, Orthopaedic Hospital of the Free University of Berlin developed an inductively powered implantable device that measures hip joint forces and temperatures for the hip joint prosthesis [50]. The temperature is measured at 10 points, and three semiconductor strain gauges sense the deformation for three-dimensional load measurement. The whole system consists of sensors and two telemetry units. Each of them is based on an eight-channel telemetry chip and transmits data at a frequency range of 140-160 MHz. An external power coil is slipped over the patient's leg to allow powering of internal coils, and its magnetic field $(4 \mathrm{KHz})$ is controlled by a personal computer. The force and temperature can be monitored in real time.

S3. Researchers at the Catholic University of Luven, Belgium, developed an implantable system for detecting the loosening of a hip prosthesis [51]. The implantable device, containing an accelerometer, a microcontroller and a transmitter, is powered by an inductive link. The accelerometer is implanted in the prosthesis' head and detects harmonics produced as a reaction to mechanical vibrations when the prosthesis becomes loose.

\subsection{Heterogeneous sensor systems}

H1. The U.S. Army Research Institute of Environmental Medicine (USARIEM) and The U.S. Army Medical Research and Materiel Command (USAMMRC) are leading the project Warfighter Physiological Status Monitoring (WPSM) $[52,53]$. The experimental system includes sensors for the heart rate, metabolic energy cost of walking, core and skin temperatures, geolocation and activity/inactivity. Data collected by various sensors are transmitted wirelessly to a hub (worn on a soldier's belt) through a low-power Personal Area Network (PAN). Sensors are expected to be low-cost and disposable, capable of collecting data for a few weeks. Aggregated data can be stored or forwarded to a warfighter's digital fighting system, command center or in the future, to the Internet. The final system is expected to be able to predict the critical aspects of performance under extreme conditions.

H2. Digital Angel Corporation [54] markets the Digital Angel system, which provides phone and e-mail alerts whenever a person or an asset changes condition or position. It also plans to add biosensors that will report pulse, 3-lead ECG, blood pressure, oxygen level, body temperature and core temperature. The system will provide physicians with a digital link to the patient that would allow them to transmit quickly bio-data in case of an emergency.

H3. The careTrend ${ }^{\mathrm{TM}}$ System, offered by Sensitron, uses a combination of Bluetooth and IEEE $802.11 \mathrm{~b}$ to send patient vital signs data from the point-of-care to the server [55]. Currently, the company offers blood pressure and pulse monitor, thermometer, weight scale, pulse oximeter and respiration rate monitor that can be wirelessly connected to the Patient Communication Unit. The caregiver can use this handheld unit to input pain scores, manage tests and communication, to select patients and to view results. The same unit is used to transfer patient data to the archiving system through the careTrends access points.

H4. Researchers at Kansas State University are developing a wearable, Bluetooth-enabled portable monitoring health system [56]. The goal is to provide affordable systems by utilizing plug-and-play sensor units that comply with one of the common industry standards for a short-range wireless communication, Bluetooth. Their current prototype comprises three units: an Internet-ready base station, a wearable data logger and a sensor unit. The system will be extended to allow multiple data loggers and multiple sensor units to be connected to each of them.

H5. Cleveland Medical Devices Inc. markets the Crystal Monitor [57] as a lightweight programmable wireless physiological monitor, capable of viewing and recording EEG, ECG, EMG, EOG and PSG signals. Collected data is wirelessly transferred to the PC, up to $1400 \mathrm{~m}$, depending on the transmitter frequency used $(150 \mathrm{kHz}-1.04 \mathrm{GHz})$ and the power the transmitter consumes $(0.01-100 \mathrm{~W})$.

H6. Thought Technology Ltd has developed a system called ProComp + [58] for real-time monitoring of EMG, EEG, EKG, skin conductance, temperature, heart rate, blood volume pulse and respiration. The device is connected to a personal computer using an optic cable, where the data can be stored and analyzed.

H7. We developed a system of intelligent sensors organized into a hierarchical network [59], which can be used for patient monitoring [60, 61, 62, 63]. Wireless connections between individual sensors and the personal monitor allow a user to freely move and to perform normal activities. Sensor intelligence allows warning generation and processing algorithm changes, while the hierarchical organization contributes to low-power consumption and processing efficiency. The system could be integrated into a wireless telemedical environment $[64,65]$.

\section{TAXONOMIES OF PERSONAL MEDICAL DEVICES}

In this section, we introduce two taxonomy groups. One group will help us classify personal medical devices based 


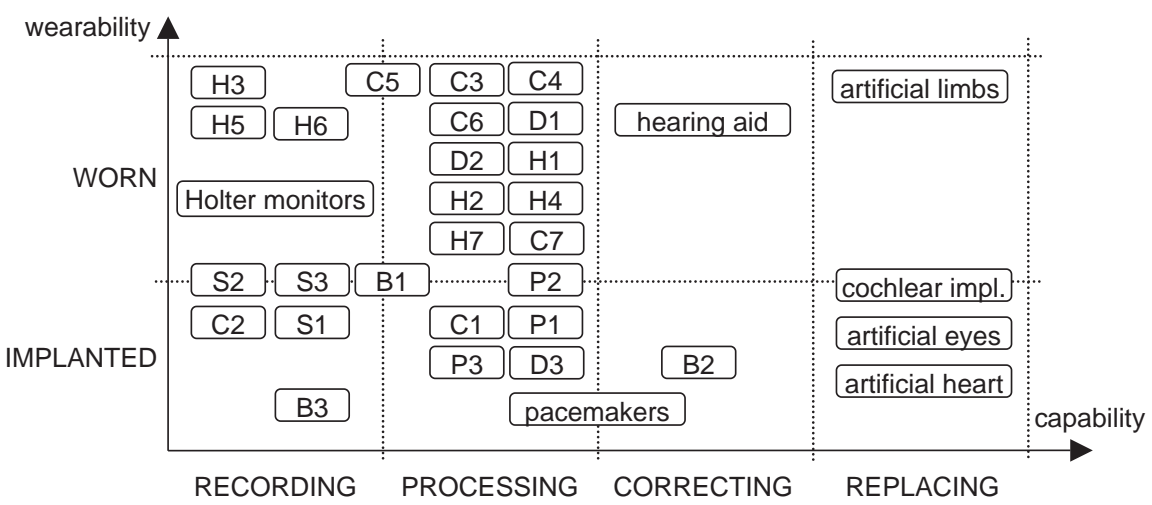

FIGURE 1. Taxonomy of usage.

upon their usage, while the other one will deal with the implementation. The taxonomies allow us to abstract from a particular application and devise design principles that hold for all applications that fall within a given category. Our taxonomy will include some devices not covered in the survey. The functionality of these devices is either well known, or goes beyond the scope of this paper: defibrillators/pacemakers [28], hearing aid devices, artificial heart [66], artificial limbs, artificial eyes [67], drug pumps etc. We include them in the taxonomy for completeness, and to show possible areas for future medical applications of wearable computing.

\subsection{Description of taxonomy of usage}

Our taxonomy of usage for personal medical devices is shown in Figure 1. The taxonomy characterizes devices along two axes: capability and mode of wearability. We see four broad categories along the capability axis: recording, processing, correcting and replacing. We define their function as:

- Recording: Devices that send or store relevant signals and data from the patient, but do not evaluate the signals (except for signal conditioning) in any manner or provide feedback to the patient. The signals are evaluated off-line, which practically disqualifies those devices from being classified as wearable computers because they perform only negligible computation.

- Processing: Devices that process relevant signals and provide immediate, conscious feedback to the patient about his or her current condition. This feedback may or may not be continuous, as in the case of an ECG monitor that provides alerts of impending cardiac events. These processing devices may also store the signals so that they may be further processed off-line, just like the recording devices.

- Correcting: Devices that provide appropriate stimuli directly to a malfunctioning organ in order to correct its behavior.

- Replacing: Devices that replace an organ entirely (prostheses).

This axis may include another category in the future, Curing. This category is somewhat related to the correcting category, but going a step further: devices that train the body in some way but then can be removed, analogous to braces for the teeth. The corrective devices currently available do not repair the body; they only make up for its shortcomings. Devices that automatically deliver a required dosage of drugs fall into the same category.

The second axis, wearability, has two possible categories: worn and implanted. By worn, we mean devices that are external to the body and are either an integral part of a garment or are worn as an accessory. Implanted devices are those that are inside the user's body. Typically, these will be surgically inserted. In some cases, however, the devices may be introduced into the body by other methods, as is the case with the wireless endoscopy capsule, which is swallowed [42, 43, 46, 47]. In the not-too-distant future, devices may be small enough to be introduced into the body by other means, such as inhalation. We include this axis because some devices straddle the border between implanted and worn, such as cochlear implants. The sensor itself is implanted but the device for taking sensor readings, processing them, providing information to the user, and (potentially) feedback to the sensor is worn.

To capture reliability and power consumption qualities, we considered a third axis, duration of required service, which could be divided into short-term, long-term and permanent. However, the addition of this third axis results in a very sparse taxonomy, because some of the purpose categories have inherent durations associated with them. For example, devices that fall into the replacing category tend to be used permanently. Short-term devices operate only for a few hours or days. Long-term devices must operate for approximately a month to a year. Permanent devices must operate for longer than a year, possibly for the patient's entire lifespan. One aspect that the duration category does not capture are devices that are meant to be worn only part of the day but over very long periods of time, such as a sleep apnea monitor. However, as stated above, the addition of this category results in a sparse taxonomy, so it was not included.

Another axis for this taxonomy was suggested by one of the reviewers, user interaction. This axis would capture the difference between systems where the active involvement of 


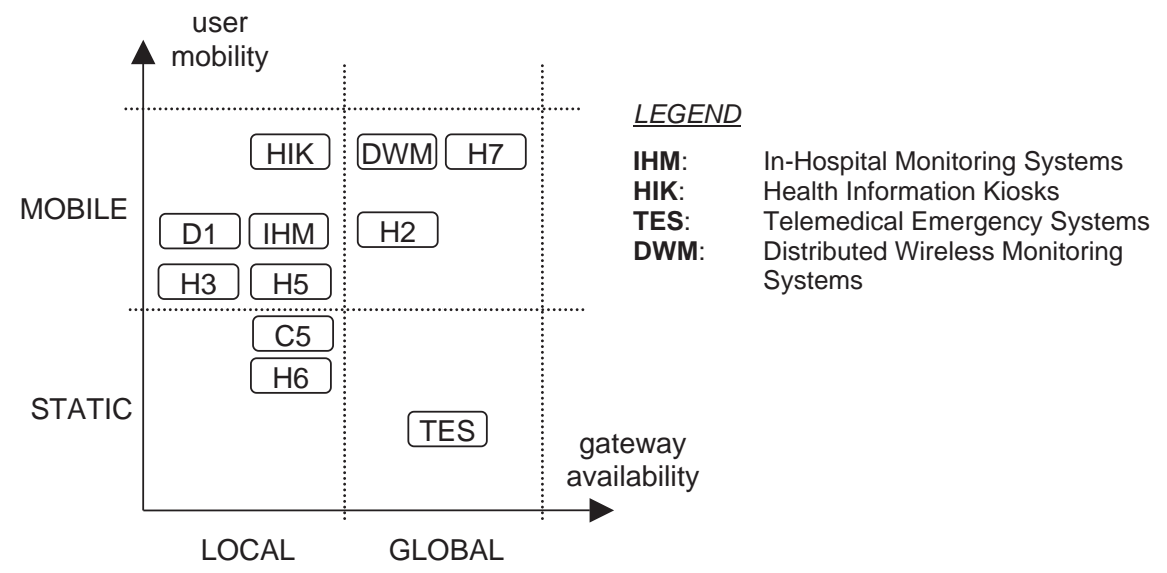

FIGURE 2. Taxonomy of implementations.

users is required as opposed to the systems (usually remote monitoring systems) where the primary user of the data collected is a caregiver.

Some devices span across certain borders in our taxonomy. For example, the system labeled as $\mathrm{P} 2$ requires an array of antennas to be worn by the patient to pick up the signal transmitted by the endoscopic pill. Therefore, it is placed on the border between worn and implanted systems. For similar reasons (powering coils), systems S2 and S3 straddle the same border in the category of recording devices. System B1 uses implanted microprobes, but the rest of the system is glued down to the back of animals. Also, some amount of processing (e.g. compression) is done on the sensor itself, and therefore this system spans across four categories in our taxonomy of capability. Finally, modern pacemakers are capable of providing statistical data about heart condition, in addition to their traditional function.

\subsection{Description of taxonomy of implementations}

Our second taxonomy is of implementations for personal medical monitors. This taxonomy classifies systems according to the system mobility (represented in Figure 2). It characterizes systems along two axes: user mobility and gateway availability. In the past, patient monitoring has been performed in a hospital or in a laboratory with the patient strapped to a fixed monitoring system and not able to move about freely. With technological advances, the monitoring equipment has become smaller, enabling wearable monitors that allowed the patient to walk around the hospital. The ultimate goal would be normal patient mobility where users are monitored as they go about their everyday routine with the monitoring equipment concealed on the person. In most cases of interest, data are not only provided to the user, but also communicated to a hospital information system or a telemedical system. Therefore, it is necessary to have an access point (gateway) to the medical network. The main system design issue is the availability of the gateway, which can be implemented as a network of fixed gateways within the medical institution, health-kiosks throughout the city, or global access point in the case of satellite-based systems. The limiting factor is the monitor power consumption necessary to maintain this connection. Because power consumption is the determining factor in the system's overall size and weight, it may be desirable to limit the distance of communication that will maintain an acceptable size of the monitoring device. Another design solution is to keep the communication distance short, but allow the access point to move around and connect with individual monitors [11]. That solution keeps the power consumption of a monitor low, at the cost of intermittent connection.

We define two broad categories along each of the axesthe patient can be either mobile or static, while the gateway availability can be either local or global. According to what we said earlier, we treat a mobile gateway as a global gateway. The ultimate goal of ubiquitous personal health monitoring systems is complete user mobility.

In our taxonomy we divide systems into four categories and provide some typical examples:

- Local Gateway/Static Patient (LGSP), typical of older monitoring systems, such as fixed bed-side hospital monitors.

- Local Gateway/Mobile Patient (LGMP), wireless inhospital or home monitoring systems [11, 55, 68, 69, 70]. This type of system allows patient mobility within the range of the network of access points. Another possible future application of LGMP is in health information kiosks that could be used to collect data from personal monitors at different locations in town.

- Global Gateway/Static Patient (GGSP), some telemedical emergency systems [71]. A typical example would include an emergency response vehicle globally connected with the medical network, while the patients are incapacitated due to the accident or illness.

- Global Gateway/Mobile Patient (GGMP) allows a patient to move freely over large areas, which is made possible by distributed wireless monitoring systems. These systems employ either cellular-phone infrastructure [72, 73] or mobile gateways as access points [19, 74]. 


\section{CONCLUSION}

Medical monitoring applications for wearable computing offer a powerful new way to keep track of a patient's medical condition and predict impending events. It can also help healthy users monitor their physiological state while working out or performing high-risk activities (military, police, firefighters etc.) or training. We have described the issues surrounding these wearable medical monitoring applications and have shown how they differ from other wearable computing applications. We have also described two taxonomies of personal medical devices to show the areas for potential uses of wearable computing and their networking requirements.

From a technical standpoint, the main obstacles to the widespread use of wearable computers for personal medical monitoring include problems with sensors, connections to the sensors and power consumption. But perhaps more difficult than the technical obstacles are the regulatory, liability and societal obstacles. Using wearable computers for medical applications will make them subject to regulation by the appropriate governmental agencies, and make manufacturers liable for the outcomes of their use. The reluctance of society to adopt systems that are technically feasible and correct will also be an obstacle, as is currently true of implantable devices. An interesting problem that arises from the overlap of technical and societal issues is that power consumption is related to privacy concerns: power consumption is a prime factor in a device's overall size and weight; if the device is too large and heavy then a user may not be able to wear it without others noticing it. Consequently, the user may not be able to keep a medical condition private because of a wearable device that is being used to monitor and/or treat the condition.

To date, the main use of wearable medical devices has been to record data that is then processed off-line. Possible nearterm future uses include not only recording the data but also processing it in real-time to provide immediate feedback to the user and, if necessary, medical personnel via a wireless connection. Particularly attractive near-term uses include devices for providing feedback during physical therapy, sports training and drug treatment. Such uses would provide medical monitoring while allowing the user to go about a normal daily routine. Longer-term uses include devices that correct or replace the functionality of some part of the user's body without having to be permanently implanted.

\section{REFERENCES}

[1] Starner, T. (2001) The challenges of wearable computing. IEEE Micro., 21(4), 44-67.

[2] Aoyagi, N., Ohashi, K., Tomono, S. and Yamamoto, Y. (2000) Temporal contribution of body movement to very long-term heart rate variability in humans. AJP-Heart and Circulatory Physiol., 7, 1035-1041.

[3] Holzman, T. (1999) Computer-human interface solutions for emergency medical care. Interactions, 6(3), 13-24.

[4] Subramanian, V. (1986) Clinical and research applications of ambulatory Holter ST-segment and heart rate monitoring. Am. J. Cardiol., 58(4), 11B-20B.
[5] Krahn, A., Klein, G., Yee, R. and Skanes, C. (1999) Recording that elusive rhythm. Can. Med. Assoc. J., 161, 1424-1425.

[6] Martin, T., Jovanov, E. and Raskovic, D. (2000) Issues in wearable computing for medical monitoring applications: a case study of a wearable ECG monitoring device. In Proc. Int. Symp. on Wearable Computers ISWC 2000, Atlanta, GA, October 18-21, pp. 43-49. IEEE Computer Society, Los Alamitos, CA.

[7] Cole, C., Blackstone, E., Pashkow, F., Snader, C. and Lauer, M. (1999) Heart-rate recovery immediately after exercise as a predictor of mortality. New Engl. J. Med., 341(18), 1351-1357.

[8] Heidenreich, P., Ruggerio, C. and Massie, B. (1999) Effect of a home monitoring system on hospitalization and resource use for patients with heart failure. Am. Heart J., 138(4), 633-640.

[9] Wheelock, B. (1999) Autonomous Real-time Detection of Silent Ischemia. MS Thesis, Electrical and Computer Engineering Department, University of Alabama in Huntsville, Huntsville.

[10] Maglaveras, N., Stamkopoulos, T., Pappas, C. and Strintzis, M. G. (1998) An adaptive backpropagation neural network for real-time ischemia episodes detection: development and performance analysis using the European ST-T database. IEEE Trans. Biomed. Eng., 45(7), 805-813.

[11] Jovanov, E., Gelabert, P., Wheelock, B., Adhami, R. and Smith, P. (2000) Real time portable heart monitoring using low power DSP. In Proc. Int. Conf. on Signal Processing Applications and Technology ICSPAT 2000 (CD Rom), Dallas, October 16-19. CMP Media LLC, Manhasset, NY.

[12] Qu, H. and Gotman, J. (1997) A patient-specific algorithm for detection of seizures onset in long-term EEG monitoring: possible use as a warning device. IEEE Trans. Biomed. Eng., 44(2), 115-122.

[13] Jung, T., Makeig, S., Stensmo, M. and Sejnowski, T. (1997) Estimating alertness from the EEG power spectrum. IEEE Trans. Biomed. Eng., 44(1), 60-69.

[14] Streitberg, B., Rohmel, J., Herrmann, W. and Kubicki, S. (1987) COMSTAT rule for vigilance classification based on spontaneous EEG activity. Neuropsychobiology, 17(1-2), 105-117.

[15] Taub, E. et al. (1999) CI therapy extended from upper to lower extremity in stroke patients. Neurosci. Abstr., 25, 320.

[16] Jannett, T. C. and DeFalque, R. J. (1990) Integrated instrumentation for closed-loop feedback control of muscle relaxation: initial clinical trials. In Proc. 12th Ann. IEEE EMBS Conf., Philadelphia, PA, November, pp. 945-946. IEEE, Piscataway, NJ.

[17] Stein, P. K. and Kleiger, R. E. (1999) Insights from the study of heart rate variability. Ann. Rev. Med., 50, 249-261.

[18] Hilton, M., Bates, R., Godfrey, K., Chappell, M. and Cayton, R. (1999) Evaluation of frequency and time-frequency spectral analysis of heart rate variability as a diagnostic marker of the sleep apnoea syndrome. Med. Biol. Eng. Comput., 37(6), 760-769.

[19] Jovanov, E., Lords, A. O. D., Raskovic, D., Cox, P., Adhami, R. and Andrasik, F. (2003) Stress monitoring using a distributed wireless intelligent sensor system. IEEE Eng. Med. Biol. Mag., 22(3), 49-55.

[20] Strub, H., Johnson, K., Allen, A., Bellotti, V. and Starner, T. (1998) Privacy, wearable computers, and recording technology (panel discussion). In Proc. Second Int. Symp. on Wearable Computers, Pittsburgh, PA, October 19-20, pp. 150-151. IEEE Computer Society Press, Los Alamitos, CA. 
[21] Bell, G. (1997) The body electric. Commun. ACM, 40(2), 31-32.

[22] University of Rochester (2003) Center for Future Health. Available at http://www.centerforfuturehealth.org

[23] Georgia Institute of Technology (2003) School of Textile and Fiber Engineering. Available at http://www.tfe.gatech.edu/.

[24] Sensatex (2003) 'Smart Shirt'. Available at http://www. sensatex.com/smartshirt/index.html.

[25] Edmison, J., Jones, M., Nakad, Z. and Martin, T. (2002) Using piezoelectric materials for wearable electronic textiles. In Proc. Sixth Int. Symp. on Wearable Computing, Seattle, WA, October 7-10, pp. 41-48. IEEE Computer Society Press, Los Alamitos, CA.

[26] Jones, M., Martin, T. and Nakad, Z. (2002) A service backplane for e-textile applications. In Proc. Workshop on Electronic Textiles, ASPLOS-X, San Jose, CA, October, pp. 15-22. ACM, New York, NY.

[27] Jung, S., Lauterbach, C. and Weber, W. (2002) Integrated microelectronics for smart textiles. In Proc. Workshop on Modeling, Analysis, and Middleware Support for Electronic Textiles (MAMSET), San Jose, CA, October 6, pp. 3-8. ACM, New York, NY.

[28] Medtronic (2003) Kappa Generation. Available at http://www. medtronic.com.

[29] CardioMEMS (2003) Cardio Micro Sensor. Available at http://www.cardiomems.com.

[30] Meridian Medical Technologies (2003) TelePulse Oximeter. Available at http://www.meridianmeds.com.

[31] Seiko (2003) Pulse Graph. Available at http://www.seikopgt.or.jp/english_k.html.

[32] Rhee, S., Yang, B.-H. and Asada, H. H. (2001) Artifactresistant power-efficient design of finger-ring plethysmographic sensor. IEEE Trans. Biomed. Eng., 48(7), 795-805.

[33] Torres-Pereira, L., Torres-Pereira, C. and Couto, C. (2000) A novel subcutaneous implantable capsule for phonocardiography. In Proc. 15th Int. Symp. on Biotelemetry, Juneau, AK, pp. 686-694. International Society on Biotelemetry, Wageningen, The Netherlands.

[34] Sontra Medical Corporation (2003) Symphony Diabetes Management System. Available at http://www.sontra.com/ productapplications/glucosemonitoring/.

[35] Morley, Jr, R. E., Richter, E. J., Klaesner, J. W., Maluf, K. S. and Mueller, M. J. (2001) In-shoe multisensory data acquisition system. IEEE Trans. Biomed. Eng., 48(7), $815-820$.

[36] Tamada, J. A., Lesho, M. and Tierney, M. J. (2002) Keeping watch on glucose. IEEE Spectrum, 39(4), 52-57.

[37] Animas Corporation (2003) Continuous Monitoring of Blood Glucose Levels. Available at http://www.animascorp. com/products/pr_glucosesensor.shtml.

[38] Diorio, C. and Mavoori, J. (2003) Computer electronics meet animal brains. IEEE Comput., 38(1), 69-75.

[39] Akin, T. and Najafi, K. (1994) A telemetrically powered and controlled implantable neural recording circuit with CMOS interface circuitry. In Proc. IEEE 7th Mediterranean Electrotechnical Conf., Antalya, Turkey, April 12-14, pp. 545-548. IEEE, New York, NY.

[40] Ghovanloo, M. and Najafi, K. (2002) A BiCMOS wireless stimulator chip for micromachined stimulating microprobes. In Proc. Second Joint EMBS/BMES Conf. (CD Rom), Houston, TX, October 23-26, pp. 2113-2114. Omnipress, Madison, WI.
[41] Irazoqui-Pastor, P., Mody, I. and Judy, J. W. (2002) Transcutaneous RF-powered neural recording device. In Proc. Second Joint EMBS/BMES Conf. (CD Rom), Houston, TX, October 23-26, pp. 2105-2106. Omnipress, Madison, WI.

[42] Astaras, A. et al. (2002) A miniature integrated electronics sensor capsule for real-time monitoring of the gastrointestinal tract (IDEAS). In Proc. ICBME 2002: The Bio-Era: New Challenges, New Frontiers, Singapore, December 4-7. Biomedical Engineering Society, Landover, MD.

[43] Aydin, N., Arslan, T. and Cumming, D. R. S. (2002) Design and implementation of a spread spectrum based communication system for an ingestible capsule. In Proc. Second Joint EMBS/BMES Conf. (CD Rom), Houston, TX, October 23-26, pp. 1773-1774. Omnipress, Madison, WI.

[44] Given Imaging (2003) M2A Capsule Endoscopy. Available at http://www.givenimaging.com.

[45] Moore, S. K. (2003) A candid camera for the gut. IEEE Spectrum, 37(7), 75.

[46] Iddan, G., Meron, G., Glukhovsky, A. and Swain, P. (2000) Wireless capsule endoscopy. Nature, 405-417.

[47] Meron, G. (2000) The development of the swallowable video capsule (M2A). Gastrointestinal Endoscopy, 52(6), 817-819.

[48] Mundt, C. W., Ricks, R. D. and Hines, J. W. (2000) Biotelemeters for space flights and fetal monitoring. In Proc. 15th Int. Symp. on Biotelemetry (Proc. Biotelemetry 15), Juneau, AK, pp. 646-651. International Society on Biotelemetry, Wageningen, The Netherlands.

[49] OAK Ridge National Lab (2003) Miniature Implantable Sensor. Available at http://www.ornl.gov/Press_Releases/ archive/mr20021122-00.html.

[50] Graichen, F., Bergmann, G. and Rohlmann, A. (2000) Implantable telemetry system for measurement of hip joint force and temperature. In Proc. 15th Int. Symp. on Biotelemetry (Proc. Biotelemetry 15), Juneau, AK, pp. 661-669. International Society on Biotelemetry, Wageningen, The Netherlands.

[51] Puers, R., Catrysse, M., Vandevoordein, G., Collier, R. J., Louridas, E., Burny, F., Donkerwolcke, M. and Moulart, F. (2000) An implantable system for detecting loosening of a hip prosthesis. In Proc. 15th Int. Symp. on Biotelemetry (Proc. Biotelemetry 15), Juneau, AK, pp. 652-660. International Society on Biotelemetry, Wageningen, The Netherlands.

[52] Hoyt, R. W., Reifman, J., Coster, T. S. and Buller, M. J. (2002) Combat medical informatics: present and future. In Proc. AMIA 2002 Ann. Symp., San Antonio, TX, November 913, pp. 335-339. American Medical Informatics Association, Bethesda, MD.

[53] Obusek, J. (2001) Warfighter physiological status monitoring. The Warrior, November/December, 6-8.

[54] Digital Angel Corporation (2003) Medical Monitoring Systems. Available at http://www.digitalangel.net/medical.asp.

[55] Sensitron (2003) The CareTrend Systems. Available at http:// www.sensitron.net/technology/theCaretrendsSystem.html.

[56] Barnes, G. E. and Warren, S. (2002) A wearable, bluetoothenabled system for home health care. In Proc. Second Joint EMBS/BMES Conf. (CD Rom), Houston, TX, October 23-26, pp. 1879-1880. Omnipress, Madison, WI. 
[57] Cleveland Medical Devices Inc. (2003) Crystal Monitor. Available at http://www.clevemed.com/products/crystal_ mon/index.html.

[58] Thought Technology Ltd. (2003) ProComp+. Available at http://www.thoughttechnology.com.

[59] Raskovic, D., Jovanov, E. and Kavi, K. (2001) Hierarchical digital signal processing. In Proc. 2001 IEEE Int. Symp. on Intelligent Signal Processing and Communication Systems (ISPACS 2001), Nashville, TN, November 20-23. IEEE, Piscataway, NJ.

[60] Jovanov, E., Price, J., Raskovic, D., Kavi, K., Martin, T. and Adhami, R. (2000) Wireless personal area networks in telemedical environment. In Proc. Third IEEE EMBS Information Technology Applications in BiomedicineWorkshop of the Int. Telemedical Information Society (ITABITIS 2000), Arlington, VA, November 9-10, pp. 22-27. IEEE, Piscataway, NJ.

[61] Jovanov, E., Raskovic, D., Price, J., Moore, A., Chapman, J. and Krishnamurthy, A. (2001) Patient monitoring using personal area networks of wireless intelligent sensors. In Proc. Biomedical Sciences Instrumentation: Proc. 38th Annual Rocky Mountain Bioengineering Symp. (RMBS 2001), Copper Mountain, CO, pp. 373-378. ISA Services Inc., Research Triangle Park, NC.

[62] Jovanov, E., Raskovic, D. and Hormigo, R. (2001) Thermistorbased breathing sensor for circadian rhythm evaluation. In Proc. Biomedical Sciences Instrumentation: Proc. 38th Annual Rocky Mountain Bioengineering Symp. (RMBS 2001), Copper Mountain, CO, April, pp. 493-497. ISA Services Inc., Research Triangle Park, NC.

[63] Milenkovic, M., Jovanov, E., Chapman, J., Raskovic, D. and Price, J. (2002) An accelerometer-based physical rehabilitation system. In Proc. 34th Southeastern Symp. on System Theory (SSST 2002), Huntsville, AL, March 18-19, pp. 57-60. IEEE, Piscataway, NJ.

[64] Jovanov, E., Starcevic, D., Samardzic, A., Marsh, A. and Obrenovic, Z. (1999) EEG analysis in a telemedical virtual world. Future Generation Comput. Syst., 15, 225-263.

[65] Starcevic, D., Obrenovic, Z., Jovanov, E. and Radivojevic, V. (2002) Implementation of virtual medical devices in Internet and wireless cellular networks. In Proc. 1st IFIP Workshop on Internet Technologies, Applications and Societal Impact, Wroclaw, Poland, October 10-11, pp. 229-242. Kluwer, Dordrecht, The Netherlands.

[66] Abiomed (2003) AbioCor Artificial Hearth. Available at http://www.abiocor.com/prodtech/Fabiocor.html.

[67] Weiland, J., Humayun, M., Liu, W. and Marco, C. D. (2000) Real-time image processing strategies for a retinal prosthesis. In Proc. DSP Technology and Education Conf. (DSPS 2000) (CD Rom), Houston, TX, August 2-4. Texas Instruments, Dallas, TX.

[68] Bai, J. (1999) A portable ECG and blood pressure telemonitoring system. IEEE Eng. Med. Biol., 18(4), 63-70.

[69] Barro, S., Presedo, J., Castro, D., Fernandez-Delgado, M., Fraga, S., Lama, M. and Vila, J. (1999) Intelligent telemonitoring of critical care patients. IEEE Eng. Med. Biol., 18(4), 80-88.

[70] Bauer, P., Sichitiu, M., Istepanian, R. and Premaratne, K. (2000) The mobile patient: wireless distributed sensor networks for patient monitoring and care. In Proc. Third Int. Conf. on Information Technology in Biomedicine, (ITAB-ITIS 2000), Arlington, VA, November 9-10, pp. 17-21. IEEE, Piscataway, NJ.

[71] Protocol (Welch Allyn) (2003) Micropaq The Ambulatory Patient Monitor. Available at http://www.monitoring. welchallyn.com/.

[72] Hung, K. and Zhang, Y. T. (2000) On the feasibility of the usage of WAP devices in telemedicine. In Proc. Information Technology Applications in Biomedicine (ITAB-ITIS 2000), Arlington, Virginia, November 9-10, pp. 28-31. IEEE, Piscataway, NJ.

[73] Hung, K. and Zhang, Y. T. (2002) Usage of Bluetooth(TM) in wireless sensors for tele-healthcare. In Proc. Second Joint EMBS/BMES Conf. (CD Rom), Houston, TX, October 23-26, pp. 1881-1882. Omnipress, Madison, WI.

[74] Jovanov, E., O’Donnel, A., Morgan, A., Priddy, B. and Hormigo, R. (2002) Prolonged telemetric monitoring of heart rate variability using wireless intelligent sensors and a mobile gateway. In Proc. Second Joint EMBS/BMES Conf. (CD Rom), Houston, TX, October 23-26, pp. 1875-1876. IEEE, Piscataway, NJ. 